\title{
Immunogenicity in Animals of a Polysaccharide-Protein Conjugate Vaccine against Type III Group B Streptococcus
}

\author{
Michael R. Wessels, ${ }^{\star \ddagger}$ Lawrence C. Paoletti, ${ }^{\star}$ Dennis L. Kasper, ${ }^{\star \star}$ José L. DiFabio, \\ Francis Michon," Kevin Holme," and Harold J. Jennings \\ *Channing Laboratory, Brigham and Women’s Hospital, and ${ }^{\ddagger}$ Division of Infectious Diseases, Beth Israel Hospital, \\ Harvard Medical School, Boston, Massachusetts 02115; and \$Division of Biological Sciences, \\ National Research Council of Canada, Ottawa, Ontario K1A OR6, Canada
}

\begin{abstract}
The native capsular polysaccharide of type III group B Streptococcus elicits a specific antibody response in only $60 \%$ of nonimmune human subjects. To enhance the immunogenicity of this polysaccharide, we coupled the type III polysaccharide to tetanus toxoid. Prior to coupling, aldehyde groups were introduced on the polysaccharide by controlled periodate oxidation, resulting in the conversion of $25 \%$ of the sialic acid residues of the polysaccharide to residues of the 8-carbon analogue of sialic acid, 5-acetamido-3,5-dideoxy-D-galactosyloctulosonic acid. Tetanus toxoid was conjugated to the polysaccharide by reductive amination, via the free aldehyde groups present on the partially oxidized sialic acid residues. Rabbits vaccinated with the conjugate vaccine produced IgG antibodies that reacted with the native type III group B streptococcal polysaccharide ( $3 / 3$ rabbits), while rabbits immunized with the unconjugated type III polysaccharide failed to respond $(0 / 3$ rabbits). Sera from animals receiving conjugate vaccine opsonized type III group B streptococci for phagocytic killing by human peripheral blood leukocytes, and protected mice against lethal challenge with live type III group B streptococci. The results suggest that this method of conjugation to a carrier protein may be a useful strategy to improve the immunogenicity of the type III group B Streptococcus polysaccharide in human subjects. (J. Clin. Invest. 1990. 86:1428-1433). Key words: immunity $\bullet$ antibody $\bullet$ sialic acid $\bullet$ capsule $\bullet$ neonatal sepsis
\end{abstract}

\section{Introduction}

Group B Streptococcus (GBS) ${ }^{1}$ is a major cause of neonatal sepsis and meningitis in the United States, with a nationwide attack rate estimated at 2-3 per 1,000 live births (1-3). Despite advances in diagnosis and improved supportive care, neonatal

A preliminary report of this work has been submitted as an abstract to the American Federation for Clinical Research national meeting, 4-7 May 1990. Issued as National Research Council of Canada No. 31895 and published in 1990 (Clin. Res. 38:393A).

Address reprint requests to Dr. Michael Wessels, Channing Laboratory, 180 Longwood Avenue, Boston, MA 02115.

Received for publication 24 January 1990 and in revised form 25 April 1990.

1. Abbreviations used in this paper: GBS, group B Streptococcus; GC-MS, gas chromatography-mass spectrometry; NMR, nuclear magnetic resonance spectroscopy.

J. Clin. Invest.

(c) The American Society for Clinical Investigation, Inc. $0021-9738 / 90 / 11 / 1428 / 06 \$ 2.00$

Volume 86, November 1990, 1428-1433
GBS infection still carries a $10-20 \%$ mortality, and an estimated $30-50 \%$ incidence of serious long term neurologic sequelae among survivors of GBS meningitis (4-6). Organisms of capsular type III are most prevalent among GBS strains causing invasive neonatal disease, accounting for approximately $60 \%$ of all GBS isolates from neonatal sepsis and meningitis cases $(2,7)$. A large body of experimental evidence has indicated that one of the major determinants of immunity to GBS infection is antibody directed against the type-specific capsular polysaccharide of the organism. Baker and Kasper demonstrated that human infants with type III GBS sepsis and meningitis uniformly had low or undetectable levels of anticapsular antibodies (8). As IgG class antibodies cross the placenta and could achieve protective levels in the fetus before delivery, immunization of women before or during pregnancy has been proposed as a strategy to prevent neonatal GBS infection $(9,10)$. Purified preparations of three of the four major capsular polysaccharides (Ia, II, III) have been given as experimental vaccines both to animals and to human volunteers (9-13). While maternal vaccination appears to be a promising approach for preventing neonatal GBS disease, the native type III polysaccharide vaccine elicits a specific antibody response in only $60 \%$ of nonimmune recipients $(10,12,13)$.

Conjugation to a carrier protein is a strategy which has been used successfully to enhance the immunogenicity of several polysaccharide antigens, including such bacterial capsular polysaccharides as the type b capsule of Haemophilus influen$z a e$, groups $\mathrm{A}$ and $\mathrm{C}$ meningococcus, and others (14-16). A variety of coupling methods have been utilized to link polysaccharides to protein carriers directly or via a synthetic spacer molecule (17). In some cases the efficiency of the coupling reaction has been increased by the introduction of functional groups at specific or random sites on the polysaccharide. Such modifications of the carbohydrate, however, may alter the structure of the polysaccharide sufficiently to affect epitope expression, in which case the polysaccharide-protein conjugate may not elicit antibodies reactive with the native antigen. Antibodies directed against the GBS type III capsular polysaccharide appear to recognize a conformational epitope. The epitope is influenced both by the charged sialic acid residues present as side chain termini of the polysaccharide, and by the polymeric state of the polysaccharide (18-20). Because of the conformational nature of the epitope of the type III polysaccharide, we sought to develop a conjugation method that would preserve both the native repeating unit structure and the conformation of the polysaccharide, yet would result in efficient coupling to a carrier protein. Treatment of the polysaccharide with controlled periodate oxidation permitted the introduction of free aldehyde groups on a portion of the side chain sialic acid residues, while preserving the native epitope. The polysaccharide was then covalently coupled to tetanus 
toxoid, via the free aldehyde groups, by reductive amination using sodium cyanoborohydride. In contrast to the purified polysaccharide alone, the polysaccharide-protein conjugate elicited high-titered IgG class antibodies in rabbits. These antibodies were opsonic in vitro, and protected mice against lethal challenge with type III GBS organisms.

\section{Methods}

Bacterial strain. GBS type III strain M781, originally isolated from a human neonate with GBS meningitis, was donated by Carol J. Baker, Baylor College of Medicine, Houston, TX. This strain was used as a source of type III GBS capsular polysaccharide, as the target strain for in vitro opsonophagocytic assays, and as the challenge strain for mouse protection assays.

Antiserum. Antiserum raised in a rabbit as described previously $(19,21)$, against formalinized whole organisms of type III GBS, was used in the ELISA inhibition assays for purification of GBS type III polysaccharide, and as a reference serum in ELISA assays for measurement of vaccine-induced antibodies.

Isolation and purification of type III GBS capsular polysaccharide. GBS type III strain M781 was grown in a dialysate $(10,000 \mathrm{~mol} w$ cutoff membrane, Pellicon Cassette System; Millipore Corp., Bedford, MA) of Columbia broth (Difco Laboratories, Inc., Detroit, MI) supplemented with glucose, $80 \mathrm{~g} /$ /iter. An 8-h culture in a volume of 6 liters was used to inoculate 60 liters of broth in a 100-liter fermentor (New Brunswick Scientific Co., Inc., Edison, NJ). The organisms were grown at $37^{\circ} \mathrm{C}$ for $16 \mathrm{~h}$, with continuous stirring $(200 \mathrm{rpm})$, aeration (5 liters/min), and $\mathrm{pH}$ maintained at 7.0 with $10 \mathrm{M} \mathrm{KOH}$. Bacterial cells were removed using a membrane filtration system (Prostak; Millipore), and the culture fluid was concentrated over a $10,000 \mathrm{~mol}$ wt cutoff pore size membrane (Pellicon) to a volume of 2 liters. The solution was brought to $30 \%$ (vol/vol) ethanol, and the precipitate formed was removed by centrifugation and discarded. Crude capsular polysaccharide was precipitated from the remaining supernatant fluid by bringing the solution to a concentration of $80 \%$ (vol/vol) ethanol. The precipitate was dried, dissolved in $10 \mathrm{mM}$ Tris- $\mathrm{HCl}, \mathrm{pH} \mathrm{7.0, \text {and }}$ treated with RNase $(250,000 \mathrm{U}$; Worthington Biochemical Corp., Freehold, NJ) and DNase (100,000 U; Worthington), then Pronase (5,000 U; Calbiochem-Behring Corp., American Hoechst Corp., San Diego, CA), each overnight at $37^{\circ} \mathrm{C}$. Contaminating group B polysaccharide was depolymerized by treatment of the solution with $1 \mathrm{~N}$ $\mathrm{NaOH}$ overnight at $37^{\circ} \mathrm{C}(22)$. The preparation was dialyzed extensively, then applied to a $2.6 \times 94 \mathrm{~cm}$ column of DEAE-Sephacel equilibrated in $10 \mathrm{mM}$ Tris- $\mathrm{HCl}, \mathrm{pH} 7.2$, and eluted with a 2-liter gradient of 0 to $0.2 \mathrm{M} \mathrm{NaCl}$ in the same buffer. Column fractions were tested by ELISA inhibition using specific rabbit antiserum for the presence of type III polysaccharide (22). Fractions showing inhibitory activity in the type III ELISA were pooled, dialyzed against water, and lyophilized. Because base treatment results in partial de- $\mathrm{N}$-acetylation of sialic acid residues on the polysaccharide, this material was re-Nacetylated by treatment with acetic anhydride, as described previously (22). Contaminating high relative molecular mass mannan (from the growth medium) was removed by passing the polysaccharide over a 2.6 $\times 28 \mathrm{~cm}$ column of Concanavalin A linked to Sepharose 4B (Sigma Chemical Co., St. Louis, MO) in $20 \mathrm{mM}$ Tris- $\mathrm{HCl}$, pH 7.4 containing $0.5 \mathrm{M} \mathrm{NaCl}, 1 \mathrm{mM} \mathrm{MnCl} 2,1 \mathrm{mM} \mathrm{CaCl}$. The polysaccharide was size fractionated by passage over a $2.6 \times 90 \mathrm{~cm}$ column of Ultragel ACA 44 (IBF Biotechnics, Inc., Savage, MD) in PBS. The material eluting at the center of the major peak was pooled, dialyzed against water, and lyophilized to yield purified capsular polysaccharide of estimated 200,000 $M_{\mathrm{r}}$. Analysis of this material by gas chromatography-mass spectrometry (GC-MS) after conversion of the component sugars to alditol acetates, and by ${ }^{1} \mathrm{H}$-nuclear magnetic resonance spectroscopy ('H-NMR), confirmed the native type III polysaccharide structure and the absence of contaminating group B polysaccharide (19).

Periodate oxidation of type III polysaccharide. GBS type III poly- saccharide ( $16 \mathrm{mg}$ ) was incubated with $4 \mu \mathrm{M}$ of sodium periodate in $1.6 \mathrm{ml}$ of water, in the dark, at $23^{\circ} \mathrm{C}$ for $1 \mathrm{~h}$. Ethylene glycol was added to consume any residual periodate, the mixture was dialyzed against water, and lyophilized. Degree of oxidation of the sialic acid residues was assessed by GC-MS of trimethylsilyl derivatives: a 1-mg sample of the oxidized polysaccharide was treated with sodium borohydride, 10 $\mathrm{mg}$ in $0.5 \mathrm{ml}$ water, at $23^{\circ} \mathrm{C}$ for $1 \mathrm{~h}$, then deionized over Rexyn $101(\mathrm{H})$ resin (Fisher Scientific Co., Fair Lawn, NJ), and evaporated three times on a rotary evaporator with 5-ml amounts of methanol. Sialic acid residues and their oxidized derivatives were hydrolyzed from the polysaccharide and converted to methyl esters/methyl glycosides by treatment with $2 \mathrm{~N}$ methanolic $\mathrm{HCl}, 1 \mathrm{ml}$, at $80^{\circ} \mathrm{C}$ for $2 \mathrm{~h}$. $\mathrm{HCl}$ was removed by evaporation with methanol, and trimethylsilyl derivatives were prepared, as described (23). Analysis of this sample by GC-MS was carried out as described (24).

Purification of tetanus toxoid. Tetanus toxoid (Institute Armand Frappier, Montreal, Canada) was purified by gel filtration chromatography before use. $15 \mathrm{ml}$ of a liquid preparation containing $1 \mathrm{mg} / \mathrm{ml}$ protein nitrogen was loaded onto a $2.6 \times 90 \mathrm{~cm}$ column of Ultragel ACA44 equilibrated in PBS and eluted with the same buffer. Protein eluted from the column in two peaks: the earlier eluting peak contained polymerized toxoid, and the later eluting peak, corresponding to $\sim 150,000 M_{\mathrm{r}}$, contained tetanus toxoid monomer. Fractions corresponding to the later (monomer) peak were pooled, dialyzed against water, and lyophilized.

Coupling of type III polysaccharide to tetanus toxoid. Periodatetreated type III polysaccharide $(11 \mathrm{mg})$ and purified tetanus toxoid monomer $(11 \mathrm{mg}$ ) were dissolved in $0.1 \mathrm{M}$ sodium bicarbonate, $\mathrm{pH}$ 8.1 . Sodium cyanoborohydride $(20 \mathrm{mg})$ was added, and the mixture was incubated at $37^{\circ} \mathrm{C}$ for $10 \mathrm{~d}$. The progress of the conjugation was monitored by removing aliquots at various time points for analysis by fast performance liquid chromatography (FPLC), using a Superose 6, HR 10/30 column (Pharmacia Fine Chemicals, Div. of Pharmacia, Inc., Piscataway, NJ), with PBS as eluant at a flow rate of $0.5 \mathrm{ml} / \mathrm{min}$. Conjugation of polysaccharide to tetanus toxoid was indicated by a progressive increase in a broad peak (monitored by measurement of UV absorbance at $214 \mathrm{~nm}$ ) eluting soon after the void volume of this column. After conjugation was complete, sodium borohydride $(10 \mathrm{mg})$ was added to the reaction mixture to reduce any remaining free aldehyde groups. The conjugate was purified by passage over a $1.6 \times 85 \mathrm{~cm}$ column of Biogel A, 0.5 M (Bio-Rad Laboratories, Richmond, CA), equilibrated in PBS, and eluted with the same buffer. Fractions corresponding to the void volume peak were pooled, dialyzed against water, and lyophilized.

Chemical analysis of the conjugate. Carbohydrate content was measured by the phenol-sulfuric acid method, using the native type III polysaccharide as a standard (25). Protein content was measured by the method of Lowry et al., using bovine serum albumin as a standard (26). Sialic acid content was measured by the resorcinol method, using sialic acid as a standard (27).

Immunization of rabbits. Female New Zealand White rabbits weighing $\sim 2 \mathrm{~kg}$ were injected with $50 \mu \mathrm{g}$ s.c. of native type III polysaccharide or $50 \mu \mathrm{g}$ of conjugate in complete Freund's adjuvant. Booster doses of $50 \mu \mathrm{g}$ of the same vaccine were given subcutaneously in incomplete Freund's adjuvant 3 and 6 wk after the first dose.

Measurement of antibodies against type III GBS polysaccharide. Antibodies against the native type III GBS polysaccharide were measured by ELISA. Native type III polysaccharide was coupled to poly-Llysine and coated onto 96-well flat bottom plastic plates at a concentration of $1 \mu \mathrm{g} / \mathrm{ml}$, as previously described (28). Test serum at several dilutions was added, and subsequent steps of addition of alkaline phosphatase coupled antirabbit IgG and substrate carried out as described previously (29). Each assay was standardized using a reference serum (raised against formalinized whole type III GBS) on the same plate. Plates were read when duplicate wells developed with the reference serum at a dilution of 1:80,000 reached absorbance at $405 \mathrm{~nm}$ $=0.5$. Antibody titer was defined as the reciprocal of the highest serum dilution giving absorbance at $405 \mathrm{~nm}>0.3$. 
Opsonophagocytic assays. Opsonophagocytic assays were performed essentially as described by Baltimore et al. (29). Early log phase organisms of type III GBS strain M781 $\left(4 \times 10^{6} \mathrm{CFU}\right)$ were incubated with $3 \times 10^{6}$ human peripheral blood leukocytes in $0.5 \mathrm{ml}$ of Eagle's minimal essential medium. Each assay contained, in addition, $10 \%$ human serum (previously absorbed with GBS type III organisms and stored at $-80^{\circ} \mathrm{C}$ to preserve complement activity) and the rabbit serum to be tested, at a final concentration of $1 \% \mathrm{vol} / \mathrm{vol}$ (i.e., 1:100 dilution). Aliquots were removed, diluted in sterile water, and spread on blood agar plates for quantitative culture at time zero and after $1 \mathrm{~h}$ of endover-end rotation at $37^{\circ} \mathrm{C}$.

Mouse protection assay. Female CD-1 mice (Charles River Breeding Laboratories, Inc., Wilmington, MA) weighing $\sim 20-25 \mathrm{~g}$ were given a single intraperitoneal injection of undiluted antiserum or antiserum diluted in PBS in a total volume of $0.2 \mathrm{ml} .24 \mathrm{~h}$ later, the mice were challenged with an $\mathrm{LD}_{90}$ dose $\left(6.6 \times 10^{7} \mathrm{CFU}\right)$ of strain $\mathrm{M} 781$, administered by intraperitoneal injection in a total volume of $1 \mathrm{ml}$ of Todd Hewitt broth. Dead animals were removed from the cages at 24-h intervals, and the overall mortality for each group was assessed at $3 \mathrm{~d}$ after challenge.

\section{Results}

Preparation of the conjugate vaccine. The presence of $\alpha(2 \rightarrow 3)$ linked sialic acid residues as side chain termini provides a unique site for introduction of free aldehyde groups on the GBS type III polysaccharide, without disrupting the native epitope. Controlled periodate oxidation results in cleavage of the polyhydroxylated tail of sialic acid, leaving the 8-carbon analogue (5-acetamido-3,5-dideoxy-D-galactosyloctulosonic acid) or 7-carbon analogue (5-acetamido-3,5-dideoxy-D-galactosylheptulosonic acid), with a free terminal aldehyde group at the site of periodate cleavage (30). Immunochemical and NMR studies of the type III GBS polysaccharide have indicated that oxidation of the sialic acid residues of the polysaccharide does not alter the antigenicity (i.e., reactivity with antibodies raised against organisms bearing the native polysaccharide) or physical conformation of the polysaccharide (18). As this modification appears not to alter the antibody binding characteristics of the native epitope, we utilized limited periodate oxidation to cleave carbon 9 from $25 \%$ of the sialic acid residues of the type III polysaccharide, leaving a free aldehyde at the carbon- 8 position of the oxidized residues available as sites for coupling to a carrier protein by reductive amination (Fig. 1). The periodate-treated polysaccharide was analyzed by GC-MS after reduction, methanolysis, and preparation of trimethylsilyl derivatives of the native or partially oxidized sialic acid residues (Fig. 2). The signals representing the native sialic acid (C9) derivative and the partially oxidized 8-carbon (C8) derivative had relative abundances of $75.5 \%$ and $24.5 \%$, respectively, confirming that $\sim 75 \%$ of the sialic acid residues remained in the native state, while $25 \%$ had been converted to the $\mathrm{C} 8$ analogue (Fig. 2). Under these conditions, further oxidation to the $\mathrm{C} 7$ analogue did not occur to an extent detectable by GC-MS analysis.

The partially oxidized polysaccharide was covalently linked to tetanus toxoid by reductive amination using sodium cyanoborohydride. The progress of the conjugation was monitored by FPLC analysis, on a Superose 6 column, of small aliquots of the reaction mixture. Polysaccharide-tetanus toxoid conjugate eluted from the column in a broad peak that began at $11 \mathrm{~min}$, just after the void volume, and extended into the beginning of a second peak (Fig. 3). The second peak, eluting at $20 \mathrm{~min}$, contained excess unconjugated tetanus tox-
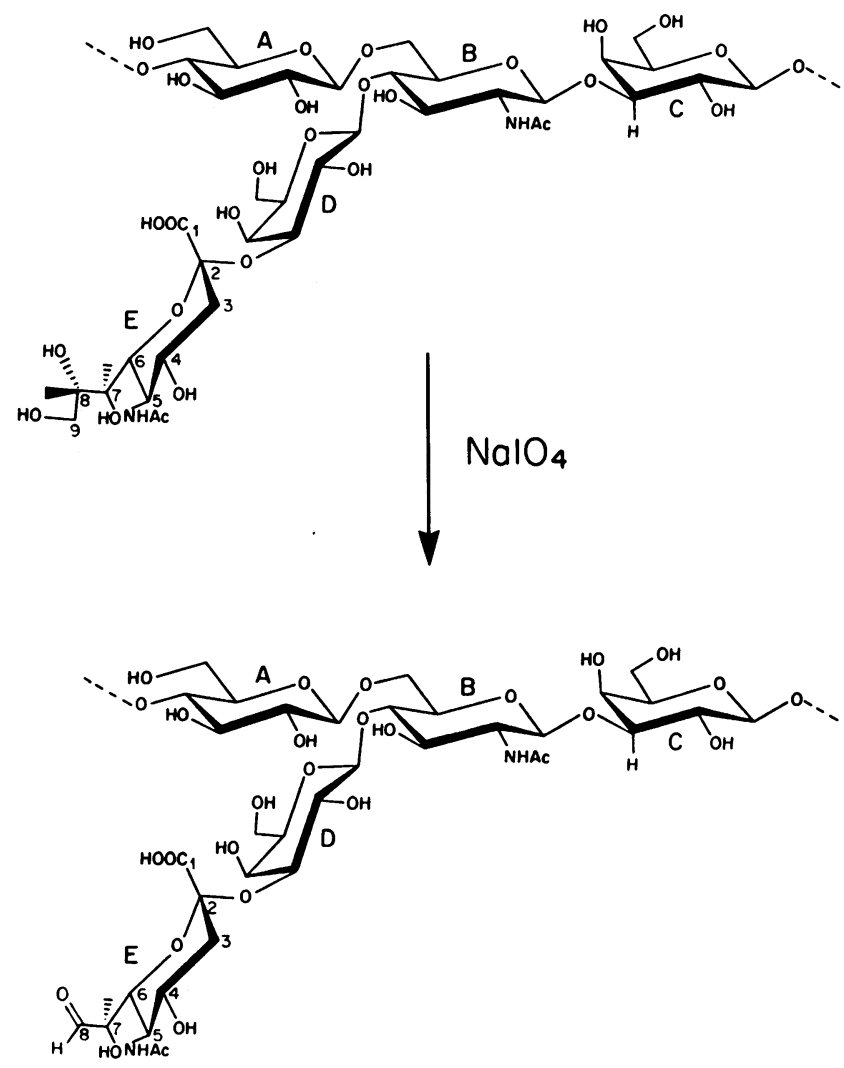

Figure 1. Limited periodate oxidation of the GBS type III polysaccharide. Approximately $25 \%$ of the sialic acid residues $(E)$ were converted to the 8-carbon analogue (5-acetamido-3,5-dideoxy-D-galactosyloctulosonic acid) with a free aldehyde group at carbon 8 .

oid (Fig. 3). After $7 \mathrm{~d}$ of incubation, no further increase was observed in the size of the first peak, indicating that conjugation was complete. The high molecular weight conjugate was purified from the reaction mixture by gel filtration chromatography on a column of Biogel A, $0.5 \mathrm{M}$. The void volume peak was collected, dialyzed against water, and lyophilized to yield $7.3 \mathrm{mg}$ of purified conjugate vaccine. The purified conjugate contained $59 \%$ protein, $41 \%$ polysaccharide, and $8 \%$ sialic acid, by weight. These values indicate that the coupled polysaccharide retained essentially the same sialic acid content as the native polysaccharide ( $20 \%$ in the coupled polysaccharide vs. $22 \%$ in the native).

Immunogenicity of the conjugate vaccine. Groups of three rabbits were vaccinated with the native polysaccharide or with the conjugate vaccine. The animals were given booster doses of the same vaccine at 3 and $6 \mathrm{wk}$ after the initial vaccination. Because the ultimate goal of a GBS type III conjugate vaccine is to induce antibodies in women which will cross the placenta, that is, IgG class antibodies, we used an IgG-specific alkaline phosphatase conjugated anti-rabbit antibody to develop the type III ELISA. None of the animals given the native polysaccharide had a rise in specific IgG antibody titer above 200 , while all three rabbits given the conjugate vaccine had increases in type III polysaccharide specific antibody titer after a single dose, which increased further after each of two booster doses (Table I). An additional group of three rabbits was vaccinated with the same dose and schedule of conjugate vaccine 


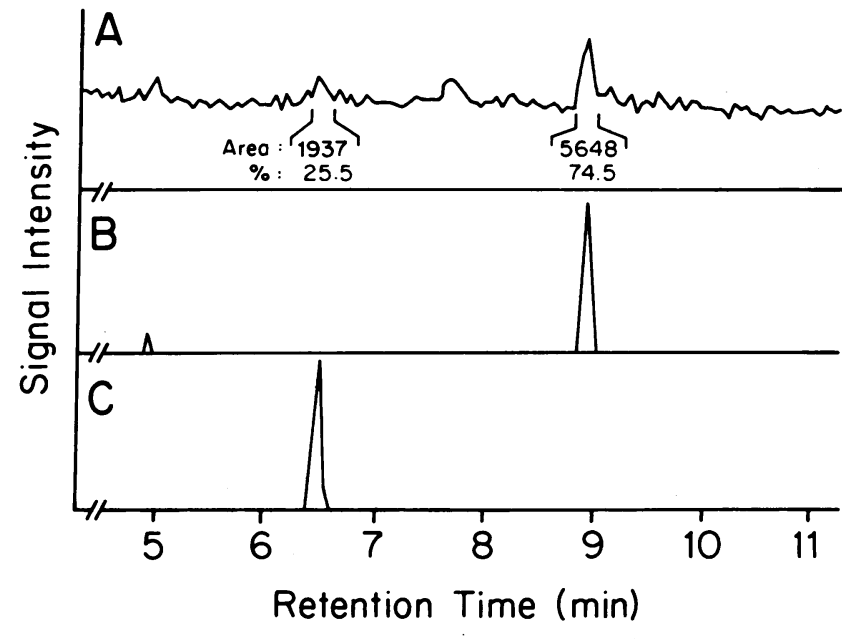

Figure 2. GC-MS analysis of periodate oxidized GBS type III polysaccharide. Residues of sialic acid (C9) and the partially oxidized 8 carbon derivative $(\mathrm{C} 8$ ) were cleaved from the polysaccharide by methanolysis to form their respective methyl esters/methyl glycosides, which were converted to trimethylsilyl derivatives for GC-MS analysis. $(A)$ Total ion current. $(B)$ Selective ion monitoring at 568 $\mathrm{m} / \mathrm{e}$ (mass corresponding to the $\mathrm{C} 9$ derivative molecular ion minus $O$-trimethylsilane). (C) Selective ion monitoring at $466 \mathrm{~m} / \mathrm{e}$ (mass corresponding to the $\mathrm{C} 8$ derivative molecular ion minus $O$-trimethylsilane). Relative abundance of the $\mathrm{C} 9$ and $\mathrm{C} 8$ derivatives is expressed as area under the curve (area) and as relative area \% (\%).

without Freund's adjuvant. Peak antibody titers of these three rabbits were $400,1,600$, and 1,600 , reached at day 34,34 , and 55 , respectively. Thus, even in the absence of adjuvant, the conjugate elicited specific IgG antibodies, while the unconjugated polysaccharide was nonimmunogenic. Sera from rabbits immunized without adjuvant were not used in opsonophagocytic assays or mouse protection studies.

Opsonic capacity of vaccine-induced antibodies. We tested the ability of antiserum from rabbits vaccinated with either the native GBS type III polysaccharide or the polysaccharide-protein conjugate vaccine to opsonize type III GBS organisms. Incubation of type III GBS with human peripheral blood leukocytes, a complement source, and sera from rabbits vaccinated with the conjugate vaccine resulted in a 1.8-2.0 log decrease in CFU after $1 \mathrm{~h}$, compared with the input inoculum (Table II). The same degree of killing was observed when a purified IgG fraction, prepared by Protein A-Sepharose affinity chromatography of conjugate-induced immune serum, was

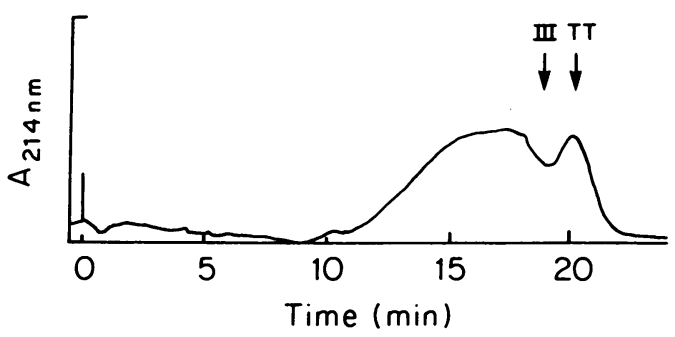

Figure 3. FPLC elution profile of conjugation reaction mixture after $7 \mathrm{~d}$ of incubation. Elution times of unconjugated GBS III polysaccharide (III) and of unconjugated tetanus toxoid (TT) are indicated by arrows.
Table I. Titers of GBS Type III Polysaccharide-Specific Antibodies, Measured by ELISA, in Sera of Rabbits Vaccinated with Native GBS Type III Polysaccharide (III Native) or with GBS Type III Polysaccharide-Tetanus Toxoid Conjugate Vaccine (III-TT)

\begin{tabular}{lrrrrr}
\hline & \multicolumn{5}{c}{ Antibody titer } \\
\cline { 2 - 6 } Vaccine & Day 0 & Day 20 & Day 34 & Day 55 & Day 75 \\
\hline III native & & & & & \\
Rabbit 1 & $100^{*}$ & 100 & 100 & 100 & 100 \\
Rabbit 2 & 100 & 100 & 200 & 100 & 100 \\
Rabbit 3 & 100 & 100 & 100 & 100 & 100 \\
III-TT & & & & & \\
Rabbit 1 & 100 & 1,000 & 16,000 & 64,000 & 128,000 \\
Rabbit 2 & 100 & 4,000 & 16,000 & 64,000 & 128,000 \\
Rabbit 3 & 100 & 500 & 32,000 & 256,000 & 256,000 \\
\hline
\end{tabular}

Values are means of duplicate determinations. Rabbits were vaccinated on days 0,20 , and $41 .{ }^{*}$ A value of 100 indicates an antibody titer of 100 or less.

substituted for whole serum (data not shown). Neither preimmune sera from rabbits receiving the conjugate vaccine, nor postvaccination sera from animals receiving the native polysaccharide produced more than a $0.15 \mathrm{log}$ reduction in CFU, under the same conditions (Table II).

Mouse protection by vaccine-induced antibodies. To further establish the functional capacity of vaccine-induced antibodies, groups of mice were injected with pooled serum from rabbits vaccinated either with the native polysaccharide or with the conjugate vaccine. $24 \mathrm{~h}$ later, the mice were given an $\mathrm{LD}_{90}$ dose of live organisms of type III GBS strain M781. Neither preimmune serum from rabbits receiving the conjugate vaccine, nor postvaccination serum from rabbits receiving the native polysaccharide alone provided any protection, while postvaccination serum from rabbits receiving the conjugate vaccine was highly protective at both doses tested: $100 \%$ sur-

Table II. Opsonophagocytic Killing of Type III GBS by Human Peripheral Blood Leukocytes in the Presence of Serum from Rabbits Vaccinated with Native GBS Type III Polysaccharide (III Native) or GBS Type III Polysaccharide-Tetanus Toxoid Conjugate (III-TT)

\begin{tabular}{lcrr}
\hline \multicolumn{1}{c}{ Serum source } & $\begin{array}{c}t=0 \mathrm{~h} \\
\text { (CFU) }\end{array}$ & \multicolumn{1}{c}{$\begin{array}{c}t=1 \mathrm{~h} \\
\text { (CFU) }\end{array}$} & Log kill \\
\hline III native, day 34 bleed & & & \\
$\quad$ Rabbit 1 & $4.8 \times 10^{6}$ & $4.4 \times 10^{6}$ & 0.03 \\
Rabbit 2 & $4.6 \times 10^{6}$ & $3.6 \times 10^{6}$ & 0.10 \\
Rabbit 3 & $4.8 \times 10^{6}$ & $5.7 \times 10^{6}$ & -0.08 \\
III-TT, prevaccination & & & \\
Rabbit 1 & $4.5 \times 10^{6}$ & $3.2 \times 10^{6}$ & 0.15 \\
Rabbit 2 & $4.7 \times 10^{6}$ & $3.5 \times 10^{6}$ & 0.13 \\
Rabbit 3 & $3.4 \times 10^{6}$ & $4.0 \times 10^{6}$ & -0.07 \\
III-TT, day 34 bleed & & & \\
Rabbit 1 & $3.3 \times 10^{6}$ & $6.8 \times 10^{4}$ & 1.7 \\
Rabbit 2 & $3.7 \times 10^{6}$ & $4.0 \times 10^{4}$ & 2.0 \\
Rabbit 3 & $3.0 \times 10^{6}$ & $4.6 \times 10^{4}$ & 1.8
\end{tabular}

Values are means of duplicate determinations. 
Table III. Survival of Mice Challenged with an $L D_{90}$ Dose of Live Type III GBS, Following Passive Immunization with Pooled Antiserum of Rabbits Vaccinated with Native GBS Type III Polysaccharide (III Native) or GBS Type III Polysaccharide-Tetanus Toxoid Conjugate (III-TT)

\begin{tabular}{lrrr}
\hline \multirow{2}{*}{\multicolumn{1}{c}{ Antiserum }} & \multicolumn{3}{c}{ No. mice surviving/no. mice challenged } \\
\cline { 2 - 4 } & Day 1 & Day 2 & Day 3 \\
\hline III native, day 34 (undiluted) & $0 / 10$ & $0 / 10$ & $0 / 10$ \\
III-TT, day 0 (undiluted) & $0 / 10$ & $0 / 10$ & $0 / 10$ \\
III-TT, day 34 (undiluted) & $10 / 10$ & $10 / 10$ & $10 / 10$ \\
III-TT, day 34 (1:10 dilution) & $10 / 11$ & $9 / 11$ & $9 / 11$
\end{tabular}

vival for the group receiving undiluted antiserum and $83 \%$ protection for the group receiving antiserum diluted 1:10 (Table III).

\section{Discussion}

A recent study by Baker et al. showed that women vaccinated in the third trimester of pregnancy with a single dose of native GBS type III polysaccharide developed IgG antibodies that were efficiently transferred to their infants (10). The major limitation of maternal immunization with the isolated polysaccharide, however, is that the type III polysaccharide elicits an antibody response in only about $60 \%$ of previously nonimmune pregnant or nonpregnant women. The results of the current study suggest that the immunogenicity of the native polysaccharide may be enhanced greatly by conjugation to a protein carrier. Bacterial polysaccharides are considered thymus independent (TI) antigens because, in contrast to thymus dependent (TD) antigens, they are able to elicit an antibody response in athymic nude mice deficient in $\mathrm{T}$ helper cells. Conjugation of a variety of polysaccharides or oligosaccharides to carrier proteins has produced immunogens with TD properties including enhanced immunogenicity, predominant IgG isotype antibodies, and a booster or anamnestic response to subsequent vaccinations $(14-16,31)$. In the case of the GBS type III polysaccharide, the goal of conjugation is simply to increase the immunogenicity of the polysaccharide antigen. In contrast to Haemophilus influenzae type b, for example, the target population for immunization with a GBS vaccine is young adults, so the delayed ontogeny of the immune response to polysaccharide antigens is not an issue. Likewise, manipulation of the immune response to induce IgG class antibodies does not appear to be necessary, as the isotype of antibody elicited by the isolated GBS type III polysaccharide in human subjects is predominantly $\operatorname{IgG}(10,32)$. Lagergard et al. have described the preparation of a GBS type III conjugate vaccine, in which the polysaccharide was activated at random sites with cyanogen bromide, then linked via a spacer molecule to tetanus toxoid (33). That conjugate elicited a greater antibody response in mice than did the native polysaccharide, but animal protection studies were not reported.

Several considerations went into the design of the conjugate vaccine used in our studies. A well characterized antigen of defined molecular size was utilized: purity of the polysaccharide was confirmed by GC and ${ }^{1} \mathrm{H}-\mathrm{NMR}$ analysis, and the saccharide used for coupling was size fractionated by gel filtration chromatography. Functional groups were introduced at defined sites on the molecule to increase the efficiency of coupling. The presence of $\alpha(2 \rightarrow 3)$ linked sialic acid residues in a terminal position on the side chains of the GBS type III polysaccharide permitted the introduction of free aldehyde groups at specific sites on the saccharide. This selective modification of the polysaccharide structure has been shown not to alter the conformational and antigenic properties of the polysaccharide (18). Coupling was done by reductive amination under mild conditions without potentially toxic spacer molecules. The efficacy of this coupling method has been demonstrated for linking other bacterial polysaccharide antigens to proteins (16). In view of potential future use in human subjects, tetanus toxoid was used as the carrier protein as this preparation has an established record of safety for immunization of pregnant women (34).

Immune defense against GBS, like that against other Gram positive bacteria, depends on clearance of the organisms by phagocytic leukocytes. Type III GBS isolates from infants with invasive GBS disease are generally resistant to phagocytic killing by peripheral blood leukocytes in the presence of nonimmune serum (29). Antibodies directed against the type III capsular polysaccharide, in concert with serum complement, effectively opsonize type III GBS for phagocytic killing, in vitro (29). The GBS type III polysaccharide-tetanus toxoid conjugate vaccine elicited high titered, functional, IgG class antibodies in rabbits. The isolated native type III polysaccharide was completely nonimmunogenic, while the conjugate vaccine elicited IgG class antibodies after a single dose. Moreover, the titer rose in all animals after each of two booster doses of conjugate vaccine given 3 and 6 wk after the first injection. The antibodies elicited by the conjugate opsonized type III GBS organisms for phagocytosis in vitro, and protected mice against lethal challenge with type III GBS organisms.

While characterization of the $T$ cell dependency of various antigens has been done primarily in mice, the rabbit model may be a better predictor of the response of human subjects to a GBS conjugate vaccine. TI antigens such as bacterial polysaccharides classically elicit a weak, predominantly IgM antibody response in mice, which cannot be augmented further by booster vaccination. In contrast, rabbits, like $40 \%$ of adult humans, uniformly fail to develop any antibody response to vaccination with the native GBS type III polysaccharide. Our data on immunogenicity of the GBS type III conjugate vaccine in rabbits suggest that conjugation of the GBS type III polysaccharide to tetanus toxoid may greatly enhance the immunogenicity of this antigen in those human subjects who fail to respond to the native polysaccharide. These results establish the immunogenicity in animals of a conjugate vaccine against type III GBS, and provide a rationale for testing a vaccine of this design in human subjects.

\section{Acknowledgments}

We thank April Blodgett and Thomas DiCesare for expert technical assistance, and Fred Cooper for the gas chromatography-mass spectrometry analysis.

This work was supported by National Institutes of Health grants AI-23339 and AI-28040, and by a grant-in-aid from the American Heart Association. J. L. DiFabio, F. Michon, and K. Holme were supported by IAF Biochem Int., Laval, P.Q., Canada. 


\section{References}

1. Institute of Medicine, National Academy of Sciences. 1985. Prospects for immunizing against Streptococcus group B. In New Vaccine Development: Establishing Priorities. Vol. I. National Academy Press, Washington, DC. 424-439.

2. Dillon, H. C., Jr., S. Khare, and B. M. Gray. 1987. Group B streptococcal carriage and diseases: a 6-year prospective study. $\mathrm{J}$. $\mathrm{Pe}$ diatr. 110:31-36.

3. Boyer, K. M., C. A. Gadzala, L. I. Burd, D. E. Fisher, J. B. Paton, and S. P. Gotoff. 1983. Selective intrapartum chemoprophylaxis of neonatal group B streptococcal early-onset disease. I. Epidemiologic rationale. J. Infect. Dis. 148:795-801.

4. Baker, C. J. 1986. Neonatal sepsis: an overview. In Clinical Use of Intravenous Immunoglobulins. A. Morell and U. Nydegger, editors. Academic Press, London. 137-144.

5. Wald, E. R., I. Bergman, H. G. Taylor, D. Chiponis, C. Porter, and K. Kubek. 1986. Long-term outcome of group B streptococcal meningitis. Pediatrics. 77:217-221.

6. Edwards, M. S., M. A. Rench, A. A. Haffar, M. A. Murphy, M. M. Desmond, and C. J. Baker. 1985. Long-term sequelae of group B streptococcal meningitis in infants. J. Pediatr. 106:717-722.

7. Baker, C. J., and F. F. Barrett. 1974. Group B streptococcal infection in infants: the importance of the various serotypes. JAMA (J. Am. Med. Assoc.) 230:1158-1160.

8. Baker, C. J., and D. L. Kasper. 1976. Correlation of maternal antibody deficiency with susceptibility to neonatal group B streptococcal infection. N. Engl. J. Med. 294:753-756.

9. Baker, C. J., and D. L. Kasper. 1985. Group B streptococcal vaccines. Rev. Infect. Dis. 7:458-467.

10. Baker, C. J., M. A. Rench, M. S. Edwards, R. J. Carpenter, B. M. Hays, and D. L. Kasper. 1988. Immunization of pregnant women with a polysaccharide vaccine of Group B Streptococcus. N. Engl. J. Med. 319:1180-1185.

11. Kasper, D. L., C. J. Baker, B. Galdes, E. Katzenellenbogen, and H. J. Jennings. 1983. Immunochemical analysis and immunogenicity of the type II group B streptococcal capsular polysaccharide. J. Clin. Invest. 72:260-269.

12. Baker, C. J., M. S. Edwards, and D. L. Kasper. 1978. Immunogenicity of polysaccharides from type III, group B Streptococcus. $J$. Clin. Invest. 61:1107-1110.

13. Eisenstein, T. K., B. J. DeCueninck, D. Resavy, G. D. Shockman, R. B. Carey, and R. M. Swenson. 1983. Quantitative determination in human sera of vaccine-induced antibody to type-specific polysaccharides of group B streptococci using an enzyme-linked immunosorbent assay. J. Infect. Dis. 147:847-856.

14. Schneerson, R., O. Barrera, A. Sutton, and J. B. Robbins. 1980. Preparation, characterization, and immunogenicity of Haemophilus influenzae type b polysaccharide-protein conjugates. J. Exp. Med. 152:361-376.

15. Anderson, P., M. E. Pichichero, and R. A. Insel. 1985. Immunogens consisting of oligosaccharides from the capsule of Haemophilus influenzae type $\mathrm{b}$ coupled to diphtheria toxoid or the toxin protein CRM197. J. Clin. Invest. 76:52-59.

16. Jennings, H. J., and C. Lugowski. 1981. Immunochemistry of group A, B, and C meningococcal polysaccharide-tetanus toxoid conjugates. J. Immunol. 127:1011-1018.

17. Dick, W. E., Jr., and M. Beurret. 1989. Glycoconjugates of bacterial carbohydrate antigens. In Conjugate Vaccines. S. Karger
A. G., Basel. Vol. 10. J. M. Cruse and R. E. Lewis, Jr., editors. Contrib. Microbiol. Immunol. 48-114.

18. Jennings, H. J., C. Lugowski, and D. L. Kasper. 1981. Conformational aspects critical to the immunospecificity of the type III group B streptococcal polysaccharide. Biochemistry USA. 20:4511-4518.

19. Wessels, M. R., V. Pozsgay, D. L. Kasper, and H. J. Jennings. 1987. Structure and immunochemistry of an oligosaccharide repeating unit of the capsular polysaccharide of type III group B Streptococcus. A revised structure for the type III group B streptococcal polysaccharide antigen. J. Biol. Chem. 262:8262-8267.

20. Wessels, M. R., A. Muñoz, and D. L. Kasper. 1987. A model of high affinity antibody binding to type III group B Streptococcus capsular polysaccharide. Proc. Natl. Acad. Sci. USA. 84:9170-9174.

21. Edwards, M. S., A. Nicholson-Weller, C. J. Baker, and D. L. Kasper. 1980. The role of specific antibody in alternative complement pathway-mediated opsonophagocytosis of type III, group B Streptococcus. J. Exp. Med. 151:1275-1287.

22. Wessels, M. R., V.-J. Benedi, H. J. Jennings, F. Michon, J. L. DiFabio, and D. L. Kasper. 1989. Isolation and characterization of the type IV group B Streptococcus capsular polysaccharide. Infect. Immun. 57:1089-1094.

23. Lifely, M. R., and F. H. Cottee. 1982. Formation and identification of two novel anhydro compounds obtained by methanolysis of $\mathrm{N}$-acetylneuraminic acid and carboxyl-reduced, meningococcal B polysaccharide. Carbohydr. Res. 107:187-197.

24. Kamerling, J. P., J. F. G. Vliegenthart, C. Versluis, and R. Schauer. 1975. Identification of O-acetylated $\mathrm{N}$-acylneuraminic acids by mass spectrometry. Carbohydr. Res. 41:7-17.

25. Dubois, M., K. A. Giles, J. K. Hamilton, P. A. Rebers, and F. Smith. 1956. Colorimetric method for determination of sugars and related substances. Anal. Chem. 28:350-356.

26. Lowry, O. H., N. J. Rosebrough, A. L. Farr, and R. J. Randall. 1951. Protein measurement with the Folin phenol reagent. J. Biol. Chem. 193:265-275.

27. Svennerholm, L. 1957. Estimation of sialic acids. II. Colorimetric resorcinol-hydrochloric method. Biochim. Biophys. Acta. 24:604611.

28. Rubens, C. E., M. R. Wessels, L. M. Heggen, and D. L. Kasper. 1987. Transposon mutagenesis of type III group B Streptococcus: correlation of capsule expression with virulence. Proc. Natl. Acad. Sci. USA. 84:7208-7212.

29. Baltimore, R. S., D. L. Kasper, C. J. Baker, and D. K. Goroff. 1977. Antigenic specificity of opsonophagocytic antibodies in rabbit antisera to group B streptococci. J. Immunol. 118:673-678.

30. Reuter, G., R. Schauer, C. Szeiki, J. P. Kamerling, and J. F. G. Vliegenthart. 1989. A detailed study of the periodate oxidation of sialic acids in glycoproteins. Glycoconjugate J. 6:35-44.

31. Seppälä, I., and O. Mäkelä. 1989. Antigenicity of dextran-protein conjugates in mice. J. Immunol. 143:1259-1264.

32. Edwards, M. S., P. A. Fuselier, M. A. Rench, D. L. Kasper, and C. J. Baker. 1984. Class specificity of naturally acquired and vaccineinduced antibody to type III group B streptococcal capsular polysaccharide: determination with a radioimmunoprecipitin assay. Infect. Immun. 44:257-261.

33. Lagergard, T., J. Shiloach, J. B. Robbins, and R. Schneerson. 1990. Synthesis and immunological properties of conjugates composed of group B Streptococcus type III capsular polysaccharide covalently bound to tetanus toxoid. Infect. Immun. 58:687-694.

34. Hayden, G. F., P. A. Sato, P. F. Wright, and R. H. Henderson. 1989. Progress in worldwide control and elimination of disease through immunization. J. Pediatr. 114:520-526. 